\title{
Cytopathological evaluation of potential malignancy of duodenal gastrinoma using aspiration smears from two patients' resected tumors (NET G1, NET G2): A case report
}

\author{
HIROTAKA NODA $^{1,2}$, KAZUYOSHI MASUDA ${ }^{1}$, MASAMI KANBARA ${ }^{2}$, \\ HIROMI MAEDA ${ }^{2}$, TADAO K. KOBAYASHI ${ }^{3}$ and CHIKAO YUTANI ${ }^{1}$ \\ Departments of ${ }^{1}$ Pathology and ${ }^{2}$ Clinical Laboratory, Amagasaki Chuo Hospital, Amagasaki, Hyogo 661-0976; \\ ${ }^{3}$ Division of Health Sciences, Cancer Education and Research Center, Osaka University \\ Graduate School of Medicine, Osaka 565-0871, Japan
}

Received October 23, 2019; Accepted February 21, 2020

DOI: $10.3892 / \mathrm{mco} .2020 .2030$

\begin{abstract}
Sporadic gastrin-producing neuroendocrine tumors (NETs) of the duodenum present with either Zollinger-Ellison syndrome or unspecific syndromes. Ki-67 scoring in cytopathology is an alternative approach for establishing the gastrinoma grade. Although the majority of NETs, including gastrinomas, occur in the duodenum, most research regarding the $\mathrm{Ki}-67$ index is focused on tumors of pancreatic origin. To the best of our knowledge, there is no study on the $\mathrm{Ki}-67$ index for cytological analysis of duodenal gastrinoma. The current report presents two cases of a 56-year-old man and a 66-year-old woman with NET G1 and G2 gastrinoma, respectively, arising in the duodenal bulb. The present report focused on the differences in nuclear pleomorphism and Ki-67 index between these two cases.
\end{abstract}

\section{Introduction}

Gastrinoma is a rare neuroendocrine tumor (NET) with an annual incidence of $0.05-0.2$ cases per 100,000 population overall (1). When Zollinger-Ellison described the eponymous syndrome (Zollinger-Ellison syndrome, ZES) in 1955 (2), the pancreas seemed the most frequent location of gastrinomas.

Correspondence to: Dr Chikao Yutani, Department of Pathology, Amagasaki Chuo Hospital, 1-12-1 Shioe, Amagasaki, Hyogo 661-0976, Japan

E-mail: cyutani@pc.zaq.jp

Abbreviations: NET, neuroendocrine tumor; ZES, ZollingerEllison syndrome; MEN1, multiple endocrine neoplasia type 1; CT, computed tomography; GI, gastrointestinal; PTH, parathyroid hormone; ICC, immunocytochemistry; IHC, immunohistochemistry; Pap, Papanicolaou; NF-PanNET, nonfunctioning pancreatic neuroendocrine tumor

Key words: gastrinoma, Ki-67, cytopathology, duodenum, immunocytochemistry
Over time, however, studies have found that gastrinoma occurs far more commonly in the duodenum than in the pancreas (2). Gastrinoma is the cause of $0.1 \%$ of peptic ulcers and $2-5 \%$ of recurrent ulcers (3). The tumors are often misdiagnosed because of their relative rarity and lack of specific symptoms. Typical symptoms include abdominal pain, secretory diarrhea, esophagitis, and hypercalcemia $(4,5)$. The prognosis of sporadic and multiple endocrine neoplasia type 1 (MEN1)-associated duodenal gastrinoma is better than that of pancreatic gastrinoma, as the former slowly progresses to metastasis (6). Surgical management is the only curative treatment for gastrinoma (7). Malignancy in gastrinoma is graded by assessing resected specimens according to the World Health Organization (WHO) 2017 criteria (1). Ki-67 scoring in cytology is an alternative approach for establishing the gastrinoma grade (8). However, the feasibility of such grading using cytopathological specimens remains unclear (1).

Cytopathological analysis of the Ki-67 index of duodenal gastrinoma has never been described previously $(9,10)$. Therefore, this study determined the optimal method of measuring the Ki-67 index and the role of cytomorphology in cytological and histopathological specimens, using resected duodenal gastrinomas as the standard criterion.

\section{Case report}

Materials. For cytological examination, to simulate the clinical practice of sampling methods by fine needle aspiration, we sampled freshly removed surgical specimens by aspiration biopsy using an $18 \mathrm{G}$ needle mounted on a syringe. Several smears were obtained which were wet fixed with $95 \%$ ethanol for at least $15 \mathrm{~min}$ and dried in cold air.

Methods. Pap and May-Grünwald-Giemsa stains were performed, and immunocytochemistry (ICC) staining was done using chromogranin A (Nichirei Biosciences Inc.), cytokeratin AE1/AE3 (Agilent Technologies Japan, Ltd.), gastrin (Agilent Technologies Japan), Ki-67 antigen (Agilent Technologies Japan), and synaptophysin (Agilent Technologies 
Table I. ICC and IHC results of Cases 1 and 2.

\begin{tabular}{|c|c|c|c|}
\hline Parameters & Case 1 & Case 2 & P-value \\
\hline Salt and pepper pattern & + & + & \\
\hline Rosette-like arrangement & + & + & \\
\hline Chromogranin A (ICC) & + & + & \\
\hline Cytokeratin AE1/AE3 (ICC) & + & + & \\
\hline Gastrin (ICC) & + & + & \\
\hline Ki-67 antigen (ICC), \% & 2 & 14 & \\
\hline $\mathrm{Ki}-67$ antigen (IHC), \% & 2 & 10 & \\
\hline Synaptophysin (ICC) & + & + & \\
\hline Mitosis & $<2 / 10 \mathrm{HPF}$ & $<2 / 10 \mathrm{HPF}$ & \\
\hline Level of tumor cell nuclear cleavage, $\%$ & 0.10 & 7.65 & $<0.001$ (tumor cells, $\mathrm{n}=954$ ) \\
\hline Mean of major axis of tumor cell nuclei, $\mu \mathrm{m}$ & 6.13 & 6.46 & $<0.001$ (tumor cells, $\mathrm{n}=486$ ) \\
\hline Mean of major axis of neurosecretory granules, $\mathrm{nm}$ & 207.94 & 427.94 & $<0.001$ (granules, $\mathrm{n}=49$ ) \\
\hline Focal margin invasion & - & + & \\
\hline Lymphovascular invasion & - & partial + & \\
\hline Histology & NET G1 & NET G2 & \\
\hline
\end{tabular}

Nuclear cleavage refers to prominent cleaved nucleus by HPF. ICC, immunocytochemistry; IHC, immunohistochemistry; HPF, high power field; NET, neuroendocrine tumor.

Japan). For dilutions in immunohistochemistry (IHC) and ICC, we used the commercial base of antibodies. Retrieval methods were followed in accordance with the IMMUNOSAVER protocol (Nissin EM Co. Ltd.).

To precisely evaluate $\mathrm{Ki}-67$ positive nuclei in both samples, 'hot spots' with more frequent positive nuclei were identified on both cytopathological and histopathological preparations. Digital images of the latter were taken, and positive nuclei were manually counted in 10 different microscopic images taken in equivalent high power magnification, (magnification, x400) microscopic fields.

For histological examination, tissues fixed in $10 \%$ buffered formalin were dehydrated and embedded in paraffin. Then, $5-\mu$ m-thick sections were cut and stained with hematoxylin and eosin and Verhoeff-van Gieson elastic stain. IHC was performed in the same manner as ICC, except deparaffinization.

For electron microscopy, specimens were immersed in a fixative containing $1.5 \%$ glutaraldehyde buffered with a $0.1 \mathrm{M}$ phosphate buffer ( $\mathrm{pH} 7.4$ ), postfixed, with osmium tetroxide, dehydrated in a graded series of ethanol baths, and embedded in epoxy resin. Ultrathin sections were cut, double-stained with uranyl acetate and lead citrate, and examined under an 80 kv H-7650 electron microscope (Hitachi).

The length of tumor cell nuclei, nuclear cleavage, and neurosecretory granules were measured using Image $\mathbf{J}$ software and then statistically compared.

Statistical analysis. All statistical analyses were performed using EZR version 1.27 (Saitama Medical Center, Jichi Medical University). Between-group differences at specific stages were assessed using Student's t-test (unpaired t-test assuming equal variances). $\mathrm{P}<0.001$ was considered statistically significant.
Case 1. A 56-year-old man was admitted to Amagasaki Chuo Hospital, Hyogo, Japan, for abdominal pain, increased serum hepatobiliary enzymes, and biliary sludge and dilatation of the common bile duct on computed tomography (CT). Upper gastrointestinal (GI) endoscopic examination showed a duodenal submucosal tumor, and incisional biopsy revealed gastrinoma. Laparoscopic partial gastrectomy around the pyloric ring was performed, and postoperative course was uneventful. Postoperatively, the patient's serum gastrin was $84 \mathrm{pg} / \mathrm{ml}$, and the serum intact parathyroid hormone (PTH) level was $37 \mathrm{pg} / \mathrm{ml}$. After 3 years of follow-up, the patient is alive and has not had any complaints. Macroscopically, the resected tumor located at the duodenal gastrinoma triangle measured $12 \times 10 \mathrm{~mm}$, with a fibrous capsule showing partial invasion into neighboring tissue. The cut surface had a lightyellow tinge similar in color to the functioning tumor's cut surface. Pap staining showed a highly cellular neoplasm with a clean background (Fig. 1). Neoplastic cells were found both in loosely cohesive clusters and as dispersed elements. The nuclei were uniform and round to oval and contained coarsely granular chromatin, referred to as a 'salt and pepper pattern.' An organoid pattern was observed, in the form of rosette-like groups, a finding that is suspicious for gastrinoma. Optically recognizable mitotic figures were not observed.

Table I shows ICC results. IHC results were identical to ICC results. Therefore, a diagnosis of gastrinoma of the duodenum, NET G1, was made (4).

Case 2. A 66-year-old woman with epigastric distress 44 months prior to admission to our hospital underwent upper GI tract-endoscopic examination; duodenal bulb biopsy revealed a NET. Chest and abdominal CT showed no particular changes; however, we detected, calcification of the 

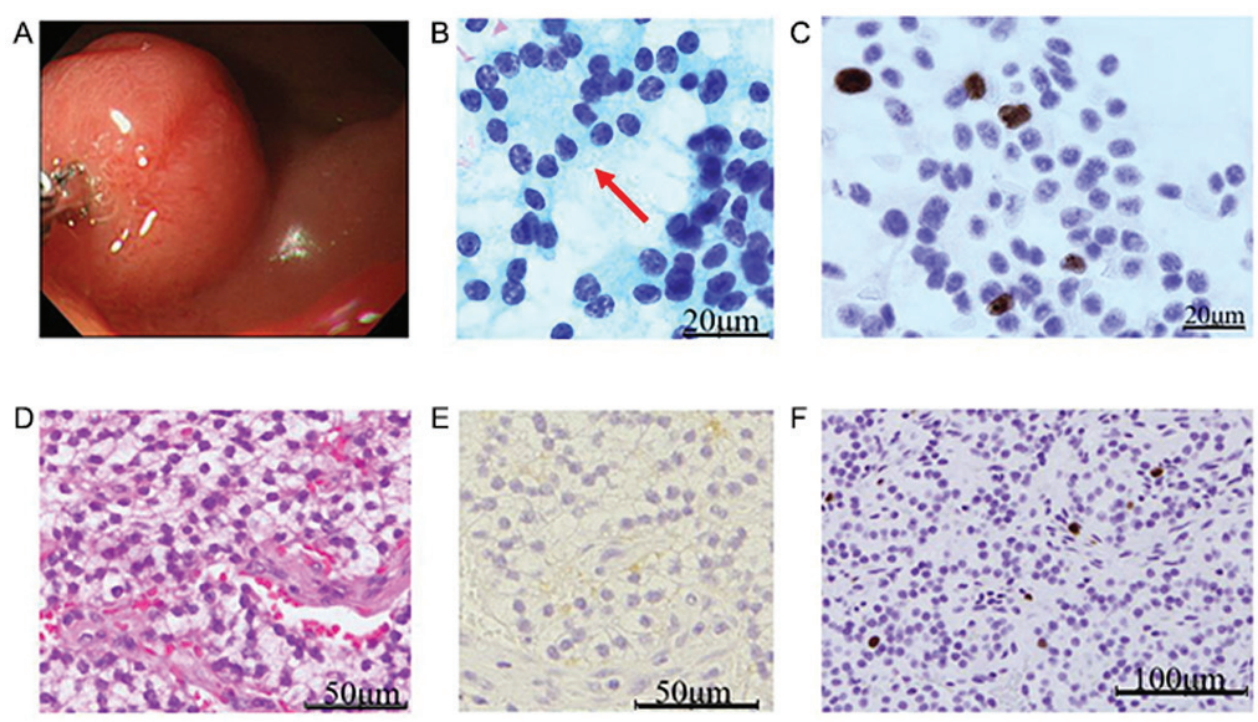

Figure 1. Case 1. (A) Endoscopic view showing duodenal gastrinoma in the gastrinoma triangle, measuring $12 \times 10 \mathrm{~mm}$ with a smooth surface and a light yellow-tinged cut surface. (B) Pap staining of a cytologic smear, showing a highly cellular organoid neoplasm with a clean background. Neoplastic cells presented as loosely cohesive clusters or as singly dispersed elements. Plasmacytoid configuration was characteristic. Uniform, round-to-oval nuclei contain coarsely granular chromatin, also defined as a 'salt-and-pepper pattern'. Rosette-like structures (arrow), characteristic of gastrinomas could also be observed. Mitotic figures were not observed (Pap stain; magnification, x1,000). (C) ICC detecting expression of Ki-67 antigen (magnification, x400). (D) Tumor cells displayed an organoid pattern, with oval-shaped nuclei and clear cytoplasms. Rosette-like arrangements could occasionally be observed. Definite atypia or mitotic figures are both rare (hematoxylin and eosin stain; magnification, x400). (E) IHC staining for gastrin (magnification, x400). (F) Positive expression of Ki-67 antigen (IHC; magnification, x400). Pap, Papanicolou; ICC, immunocytochemistry; IHC, immunohistochemistry.
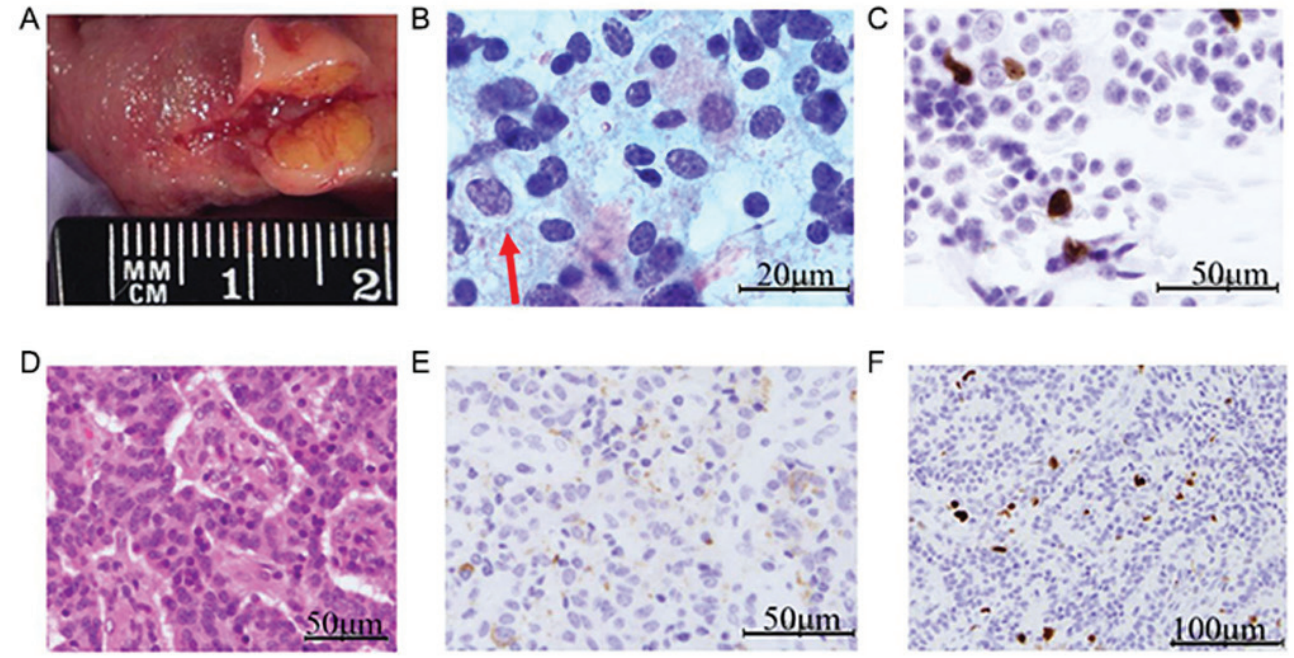

Figure 2. Case 2. (A) Submucosal tumor of the duodenal bulb measuring 10x10x7 mm with a light-yellow-tinged cut surface. (B) Pap staining revealed that tumor cells were arranged in loosely cohesive acinar clusters with slightly coarse cytoplasmic granules. Tumor cell chromatin exhibited a salt-and-pepper or dispersed arrangement. Nuclear atypia (arrow) was present but mitotic figures were rare (magnification, x400). (C) ICC for expression of Ki-67 antigen (magnification, x400). (D) Tumor cells form trabecular, glandular or mixed structures. The tumor capsule/pseudocapsule was relatively well preserved, with partial lymphovascular invasion. Rosette-like structures were occasionally visible and mitotic figures were rare (hematoxylin and eosin; magnification, $\mathrm{x} 400$ ). (E) IHC for expression of gastrin (magnification, x400). (F) IHC for expression of Ki-67 antigen (magnification, x400). Pap, Papanicolou; ICC, immunocytochemistry; IHC, immunohistochemistry.

right thyroid lobe. Cystic lesions of the liver and kidney were also observed, while cranial magnetic resonance imaging did not reveal pituitary adenoma. Her parathyroid glands showed no swelling. Abdominal exploratory subtotal resection of the stomach including the pyloric ring, was performed, using the Roux-en-Y method. The patient's serum gastrin was $684 \mathrm{pg} / \mathrm{ml}$ before surgery and $80 \mathrm{pg} / \mathrm{ml}$ after surgery, and her serum intact PTH level was $15 \mathrm{pg} / \mathrm{ml}$. After 2 years of follow-up, the patient has not complained of GI tract symp- toms. The submucosal tumor at the duodenal bulb measured 10x10x7 mm, and its cut surface, too, revealed a light-yellow tinge. Pap staining of the obtained smears showed tumor cells arranged in loosely cohesive clusters or individually; both contained slightly coarse cytoplasmatic granules. The tumor cell chromatin was finely granular and showed a salt-and-pepper or focally dispersed pattern. Nuclear atypia was present, but mitotic figures were rare (Fig. 2). Table I shows ICC results. The tumor capsule/pseudocapsule showed 

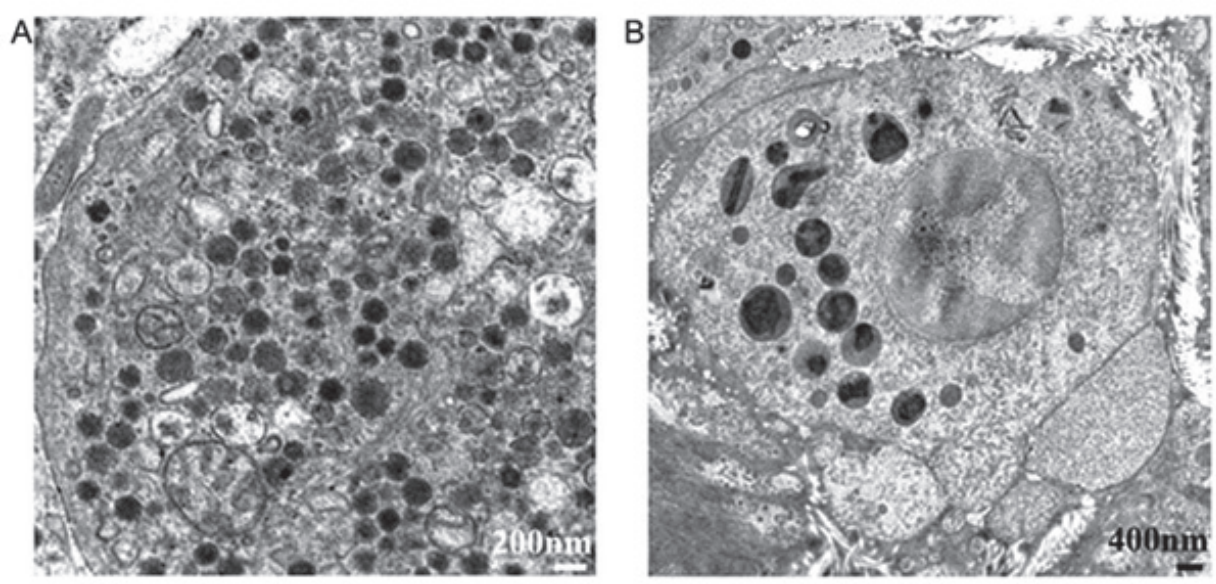

Figure 3. Electron microscopy images. A variable number of membrane-bound electron-dense neurosecretory granules were found, either nonpolarized within the cytoplasm or oriented near the basal surfaces facing the capillaries. Granule morphology was nonspecific. (A) Case 1, NET G1. (B) Case 2, NET G2. NET, neuroendocrine tumor.

focal invasion but was mostly intact, while partial lymphovascular invasion was observed. IHC results were similar to ICC results. Therefore, a diagnosis of gastrinoma of the duodenum, NET G2, was made. Table I also shows comparisons of the Ki-67 index and nuclear cleavage between Cases 1 and 2. The Ki-67 indexes of Cases 1 and 2 were cytologically 2 and $14 \%$, respectively. Alternatively, histopathologically they were 2 and $10 \%$, respectively. The optical mitotic count was $<2 / 10$ high power fields (HPF) in both cases. The tumor cell nuclear cleavage level was statistically significant $(\mathrm{P}<0.001 ; \mathrm{n}=954)$ between Cases 1 and 2 . In addition, the major axis of tumor cell nuclei was statistically significant $(\mathrm{P}<0.0010 .001 ; \mathrm{n}=486)$ between Cases 1 and 2 (Table I). We found variable numbers of membrane-bound electron-dense neurosecretory granules (Fig. 3), either nonpolarized within the cytoplasm or oriented near the basal surfaces facing the capillaries. The major axis of neurosecretory granules on ultrastructural findings was statistically significant $(\mathrm{P}<0.001$; $\mathrm{n}=49$ ) between Cases 1 and 2 (Table I).

\section{Discussion}

We found no remarkable differences in clinical characteristics between Cases 1 and 2; that is, neither tumor revealed ZES or MEN1 findings. Differences between Cases 1 and 2 were observed not only at the serum gastrin level but also in the following cytopathological findings. Among cytological findings, the $\mathrm{Ki}-67$ index, the nuclear cleavage level, the major axis of tumor cell nuclei, and the major axis of neurosecretory granules in Case 1 were lower than in Case 2. Among histopathological findings, focal margin invasion by tumor extension and lymphovascular invasion were observed in Case 2 but not in Case 1 (Table I).

With regard to the development and differentiation of gastrinoma cells, Imamura et al (11) have reported that duodenal gastrinomas do not seem to behave in as malignant a fashion as sporadic pancreatic gastrinomas. However, this report does, not validate this difference. On the basis of the $\mathrm{Ki}-67$ index as a potential prognostic marker, we also applied the WHO Ki-67 labeling scheme for grading on cytological samples. The two cases in this study indicated that cytologic samples from duodenal gastrinomas can be accurately graded on the basis of the WHO Ki-67 labeling scheme, indicating that $\mathrm{Ki}-67$ scoring of cytology preparations is an alternative approach for establishing the pancreatic NET (PanNET) grade, as shown by Farrell et al (8).

As stated by Adsay (12) in an editorial in the American Journal of Surgical Pathology, the question is no longer whether to count $\mathrm{Ki}-67$ in gastrointestinal and pancreatobiliary tract NETs; rather, it is how to perform this count and how to improve the diagnostic and prognostic value of this proliferation marker (12). However, the new WHO classification states, that mitotic figures are not usually seen in gastrinomas and that nonfunctioning pancreatic neuroendocrine tumor (NF-PanNET) grading on the basis of cytological specimens is not well established (1). The data presented here revealed that mitotic figures are not contributory for the evaluation of potential malignancy; rather, the $\mathrm{Ki}-67$ index is really contributive.

According to Chatzipantelis et al (9), with regard to cytopathological findings, nuclear pleomorphism/multinucleation and the presence of nucleoli are also reliable for predicting malignant PanNETs. Therefore, we also considered nuclear pleomorphism as a criterion suggestive of potential malignancy. Our findings revealed a positive relationship between nuclear pleomorphism and the Ki-67 index.

In recent years, numerous suggestions have been made to predict the biological behavior of these tumors. The most recent WHO classification includes several clinicopathologic criteria: Tumor size, metastasis likelihood, vascular invasion, necrosis, status of regional lymph nodes, tumor grade, mitotic rate, and $\mathrm{Ki}-67$ index (1).

In conclusion, this study demonstrates the role of proliferative activity (Ki-67) in cytology specimens as a reliable predictive factor for duodenal gastrinomas. In addition, specific cytologic features such as nuclear cleavage and nuclear major axis can contribute to the prediction of these tumors. However, because of the limited number of cases here (two), large series will be needed to confirm our findings. 


\section{Acknowledgements}

Not applicable.

\section{Funding}

No funding was received.

\section{Availability of data and materials}

The datasets used and/or analyzed during the present study are available from the corresponding author on reasonable request.

\section{Authors' contributions}

The present study was designed and organized by HN, KM, MK, HM, CY and TKK. Clinical data were corrected by HN and CY. Immunostaining was evaluated by HN. HN, CY and TKK contributed to data analysis, interpretation and drafting manuscript. All authors read and approved the final manuscript.

\section{Ethics approval and consent to participate.}

Ethical approval for the study was obtained from the Ethics Committee of Amagasaki Chuo Hospital and the two patients provided informed consent.

\section{Patient consent for publication}

Written informed consent was obtained from the two patients for publication of the clinical data and any accompanying images.

\section{Competing interests}

The authors declare that they have no competing interests.

\section{References}

1. Lloyd RV, Osamura RY, Klöppel G and Rosai J: WHO classification of tumours of endocrine organs 4th edition, Lyon, France: IARC Press: pp 229-232, 2017
2. Zollinger RM and Ellison EH: Primary peptic ulcerations of the jejunum associated with islet cell tumors of the pancreas. 1955. CA Cancer J Clin 39: 231-247, 1989.

3. Rosentraeger MJ, Garbrecht N, Anlauf M, Raffel A, Knoefel WT, Wiedenmann B and Klöppel G: Syndromic versus non-syndromic sporadic gastrin-producing neuroendocrine tumors of the duodenum: Comparison of pathological features and biological behavior. Virchows Arch 468: 277-287, 2016.

4. Roy PK, Venzon DJ, Shojamanesh H, Abou-Saif A, Peghini P, Doppman JL, Gibril F and Jensen RT: Zollinger-Ellison syndrome. Clinical presentation in 261 patients. Medicine (Baltimore) 79: 379-411, 2000.

5. Zhang WD, Liu DR, Wang P, Zhao JG, Wang ZF and Chen LI: Clinical treatment of gastrinoma: A case report and review of the literature. Oncol Lett 11: 3433-3437, 2016.

6. Anlauf M, Garbrecht N, Henopp T, Schmitt A, Schlenger R, Raffel A, Krausch M, Gimm O, Eisenberger CF, Knoefel WT, et al: Sporadic versus hereditary gastrinomas of the duodenum and pancreas: Distinct clinic-pathological and epidemiogical features. World J Gastrenteterol 12: 5440-5446, 2006.

7. Norton JA, Fraker DL, Alexander HR, Venzon DJ, Doppman JL, Serrano J, Goebel SU, Peghini PL, Roy PK, Gibril F and Jensen RT: Surgery to cure the Zollinger-Ellison syndrome. N Engl J Med 341: 635-644, 1999.

8. Farrell JM Pang JC, Kim GE and Tabatabai ZL: Pancreatic neuroendocrine tumors: Accurate grading with Ki-67 index on fine-needle aspiration specimens using the WHO 2010/ENETS criteria. Cancer Cytopathol 122: 770-778, 2014.

9. Chatzipantelis P, Konstantinou P, Kaklamanos M, Apostolou G and Salla C: The role of cytomorphology and proliferative activity in predicting biological behavior of pancreatic neuroendocrine tumors: A study by endoscopic ultrasound-guided fine-needle aspiration cytology. Cancer 117: 211-216, 2009.

10. Klöppel G and Anlauf M: Gastrinoma-morphological aspects. Wien Klin Wochenschr 119: 579-584, 2007.

11. Imamura M, Kanda M, Takahashi K, Shimada Y, Miyahara T, Wagata T, Hashimoto M, Tobe T and Soga J: Clinicopathological characteristics of duodenal microgastrinomas. World J Surg 16: 703-709, 1992.

12. Adsay V: Ki67 labeling index in neuroendocrine tumors of the gastrointetinal and pancreatobiliary tract: To count or not to count is not the question, but rather how to count. Am J Surg Pathol 36: 1743-1746, 2012. 